\title{
A TARGET AWARE TEXTURE MAPPING FOR SCULPTURE HERITAGE MODELING
}

\author{
C. Yang a, b, F. Zhang a,d, X. Huang a,c,d *, D. Li ${ }^{\text {a }}$, Y. Zhu ${ }^{\text {a }}$ \\ ${ }^{a}$ LIESMARS, Wuhan University, Wuhan, China - (, huangxf, zhangfan, drli)@ whu.edu.tr \\ ${ }^{\mathrm{b}}$ School of Remote Sensing and Information Engineering, Wuhan University, Luoyu Road 129, Wuhan, China \\ ${ }^{c}$ Collaborative Innovation Center of Geospatial Technology, Wuhan University, Luoyu Road 129, Wuhan, China \\ ${ }^{\mathrm{d}}$ Laboratory of Digital Yungang, Datong, China
}

\section{Commission VI, WG VI/4}

KEY WORDS: Texture Mapping, Silhouette, Laser Scanning, Modelling

\begin{abstract}
:
In this paper, we proposed a target aware image to model registration method using silhouette as the matching clues. The target sculpture object in natural environment can be automatically detected from image with complex background with assistant of 3D geometric data. Then the silhouette can be automatically extracted and applied in image to model matching. Due to the user don't need to deliberately draw target area, the time consumption for precisely image to model matching operation can be greatly reduced. To enhance the function of this method, we also improved the silhouette matching algorithm to support conditional silhouette matching. Two experiments using a stone lion sculpture of Ming Dynasty and a potable relic in museum are given to evaluate the method we proposed. The method we proposed in this paper is extended and developed into a mature software applied in many culture heritage documentation projects.
\end{abstract}

\section{INTRODUCTION}

\subsection{Background}

Sculptures are one of the most important forms of cultural heritage with complex geometric structure and intricate texture information (Fontana et al. 2002). Producing high precision 3D digital sculpture models is a primary work for cultural heritage protection and visualization (Ikeuchi et al. 2007; Chow and Chan 2009). Laser scanning is a reliable and efficient way, which utilizes structured light or time of flight (TOF) technique to acquire high precision geometric information (Pavlidis et al. 2007). Though camera is settled to some laser scanner system, additional images collected from elaborately selected view angle and fine light condition are necessary to achieve high fidelity texture reconstruction (Andrade et al. 2012).

In order to create model with high fidelity texture, a precise image to geometric model mapping is a necessary step. For sculptures with complex structure, this work will be challenging task (Arbace el al. 2012). Texture mapping is a kind of $2 \mathrm{~d}$ image to $3 \mathrm{~d}$ model registration problem. The key point of this problem is finding correspondence between two data sources. Usually, a few pairs of control points are collected from image and geometric model to solve the image orientation parameters, as well as inner parameters. Image can be precisely mapped to model using the orientation parameters. This method works well for single image to geometric model registration. Sometime, there are a bundle of images with overlap enough to satisfy photogrammetry requirements. Then, texture to model relation can be solved by photogrammetric or computer vision solution (Tzur and Tal , 2009).

However, for many sculptures, it is not easy to pick control points precise enough to create accurate parameters by solving the collinearity equations, thus resulting to mis-matching problem. Silhouette matching is another way for image to model registration which was applied to texture mapping in (Hendrik $P$. A. et al., 2001). But this method requires that the image should has very clean background so as to easily detect the silhouette lines. It is suitable for processing texture mapping of portable sculptures in the museum, where it is easy to set up a single colour background while collecting images. For the sculpture in natural environment or importable objects, this method cannot be applied, due to the requirement of clean background image cannot be satisfied. In many modelling cases, the sculpture texture images that collected by photographer contain both target object information and complex background information. Intelligently extract the target area from source texture image and mapping it to geometric model could greatly save processing time.

Figure 1 (a) shows a stone lion sculpture of Ming Dynasty in the natural environment with grass and bushes around. Figure 1 (b) is $3 \mathrm{~d}$ geometric model of this stone sculpture created using Geomagic software. The point cloud data was collected using $\mathrm{Z}+\mathrm{F}$ imager 5006 laser scanner from different angles. During geometric model editing processing, we removed all the noise points from laser scanner and only kept the data of sculpture.
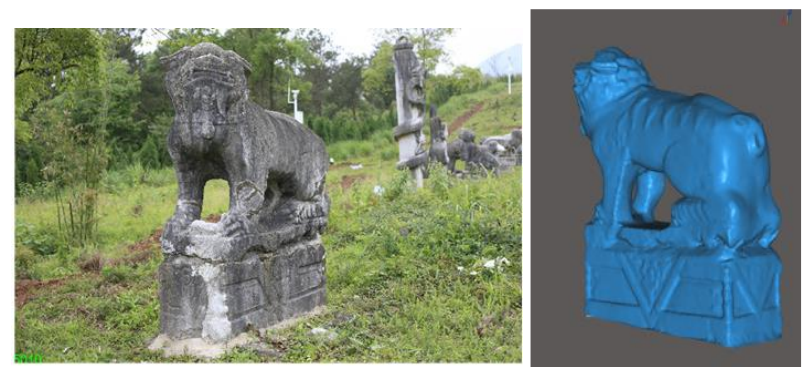

Figure 1. Test Dataset I: a stone lion in natural environment. (a) Image of stone sculpture collected by Canon camera; (b) geometric model.

To solve the texture mapping problem, we proposed a target aware image to model registration method using silhouette as the matching clues. User only need to put geometric model close to the target area in image, the silhouette can be automatically extracted and applied in image to model matching. Due to the user don't need to deliberately draw target area, the time

\footnotetext{
* Corresponding author: Xianfeng Huang, prof. of LIESMARS, Wuhan University.
} 
consumption for precisely image to model matching operation can be greatly reduced.

The paper is organized as following: In the second section, methodology will be explained. The method of target area detection and one-cut optimization will be discussed in this section. The second part of this section explains the procedure of texture to model matching using silhouette information. In section three, two experiments will be introduced with analysis to evaluate the effectiveness of this algorithm. And, a detailed discussion will be given at the end.

\section{METHODOLOGY}

\subsection{Overview}

In this study, we assume that precise geometric model has been collected using handhold laser scanner or structure lights system. The goal of this study is to reduce user interaction in texture mapping processing, especially in identifying the target.

As we know, fully automatic target detection without any predefined knowledge is a very challenging task. To simplify this problem, we utilize $3 \mathrm{~d}$ geometric model as the clue for target detection. We developed a user interface using OpenGL which is able to show texture image and $3 \mathrm{~d}$ geometry data simultaneously. User can move $3 \mathrm{~d}$ model to image close to the target area. Then, target detection using one-cut algorithm will be applied to find the target area, and sketch precise boundary of detected target area. The boundary of target is finally utilized as features for texture to model registration, using silhouette texture registration algorithm.

\subsection{Target Detection using One-Cut}

To decrease the level of difficulty, we ask the user put $3 \mathrm{~d}$ model to image where the target located at. To make this task simple enough and can be finished in a few second, we visualize the $3 \mathrm{~d}$ geometric model in $3 \mathrm{~d}$ space, as well as image. From OpenGL system, we can find $3 \mathrm{~d}$ model area and extract silhouette information using depth image in OpenGL visualization buffer. Since we don't require user giving precise position, the operation can be done with very short time.

2.2.1 Experiment Environment: In OpenGL system, the final visualization result in the screen can be seemed as a photo comes from projection of $3 \mathrm{~d}$ model to plane. If we rotate the model, we can get the depth image of each frame with given $3 \mathrm{~d}$ model, according to the camera parameters in OpenGL. Figure 2 shows the projection relation between $3 \mathrm{~d}$ model and frustum. If we change the parameters of OpenGL, the depth image will change precisely follow the parameter we give. Figure 3 is the results of put geometric model into image in our experimental $3 \mathrm{~d}$ visualization system.

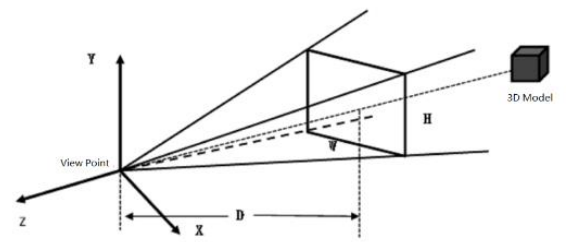

Figure 2. Camera system of OpenGL.

In Figure 2:

$\mathrm{W}, \mathrm{H}$ is the width and height of $3 \mathrm{~d}$ depth image.

$\mathrm{D}$ is the distance of view point to culling plane.

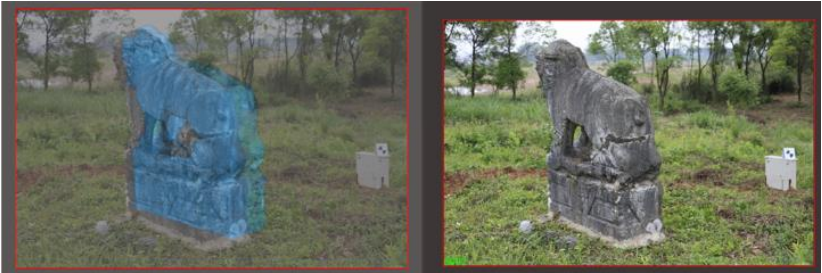

Figure 3. Overlap effect of 3d model with image.

2.2.2 Seed Area Extraction: For a given $3 d$ model in the depth image, the background area has a same invalid value, while the front parts contain meaningful number. We make the depth image into a binary image to find model block. It is very easy to find the target area in depth image, leading to a result as shown in Figure 4.

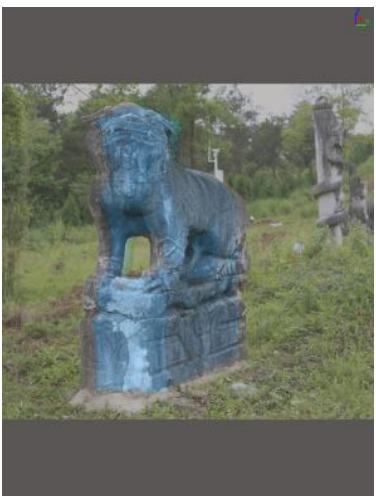

(a)

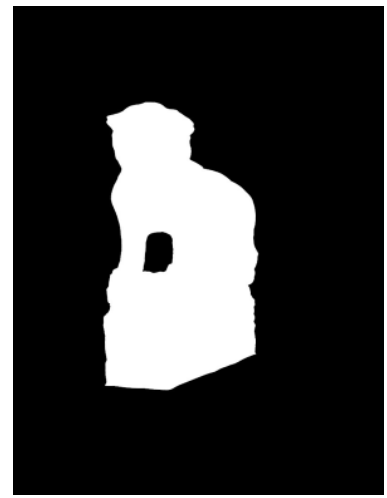

(b)
Figure 4. Target block area in depth image from 3d model.

For simple object, we can combine this binary image with colourful texture image to find out the target area, denoted in figure 4 white area as front and black as back ground. But the $3 \mathrm{~d}$ model sometimes contains a few holes, using the binary image block directly to label the texture image into front and back is not sufficient. Meanwhile, 3d model cannot overlap all the target image area for we didn't require a precise $3 \mathrm{~d}$ model position setting.

To make the algorithm robustly processing complex situation, a post processing using erosion operation is conducted, as shown in Figure 5. Two steps are involved in our binary image processing: (1) apply erosion operation at different level to binary image, getting two result images (left, middle); (2) apply exclusive-or to the two result images and get result (right) as the seed area for target detection.
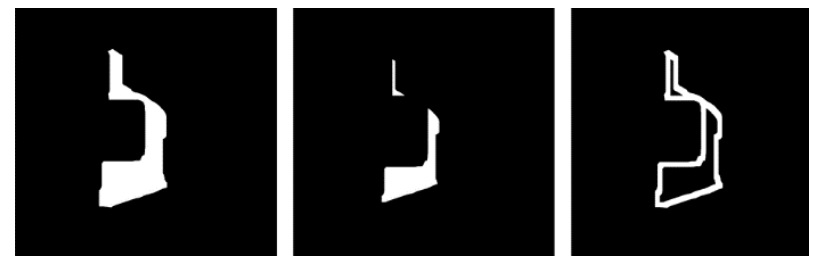

Figure 5. Target Seed Area Extraction from 3D data.

2.2.3 Seed Background Extraction: Since the background area is larger than target area, we need to find area outside the model boundary as background. 
In corresponding to target seed area extraction, an expanding algorithm is applied to the binary image to get a bigger background area. Similar to target seed area detection, two different level expanding operations and a consequent exclusiveor operation are applied to the binary image. In Figure 6, left and middle images are the results of expanding operation and right is the final background seeds area.
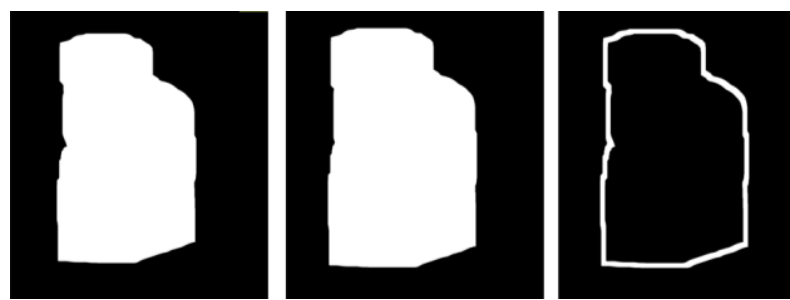

Figure 6. Seed Background Extraction using Expanding Operation.

According to the abover section, the seed of target area and background area are extracted. Combining the seed area to texture image, it is clearyly shown that target area and background area correctly surround the target sculpture (in Figure 7). The blue line means area that contain background, while read line cover front part, means target. The blue line and red line both are seed area.

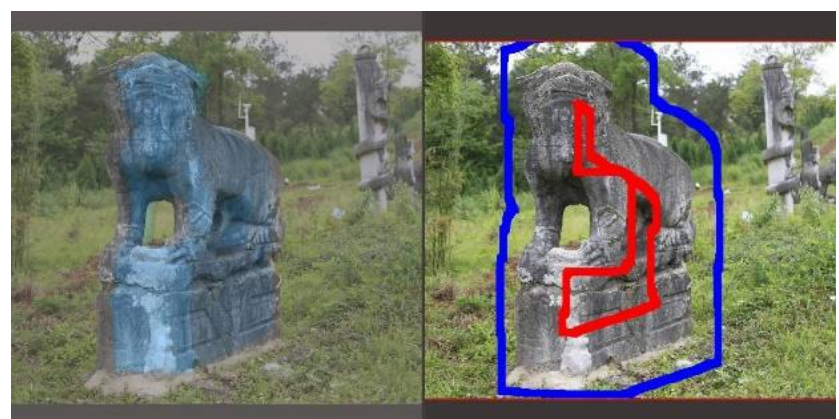

Figure 7. Target seed and background seed surround the target sculpture.

2.2.4 Target Optimization using One-Cut: Graph based algorithm is a powerful tool for image segmentation, especially for find object from background. Graph cut is one the classic graph based image segmentation algorithms (Boykov and Jolly, 2001).

Graph based image segmentation method normally change image segmentation into a min-cut problem in graph theory. An image can be seemed as a weight graph $\mathrm{G}=\langle\mathrm{P}, \mathrm{B}\rangle, \mathrm{P}$ is the set of nodes in graph, while B is set of edges. The rule of segmentation is keeping minimum similarity between sub-graphs, but maximum similarity inside each sub-graphs. The essence of graph cut algorithm is breaking unstable or weak edges inside graph.

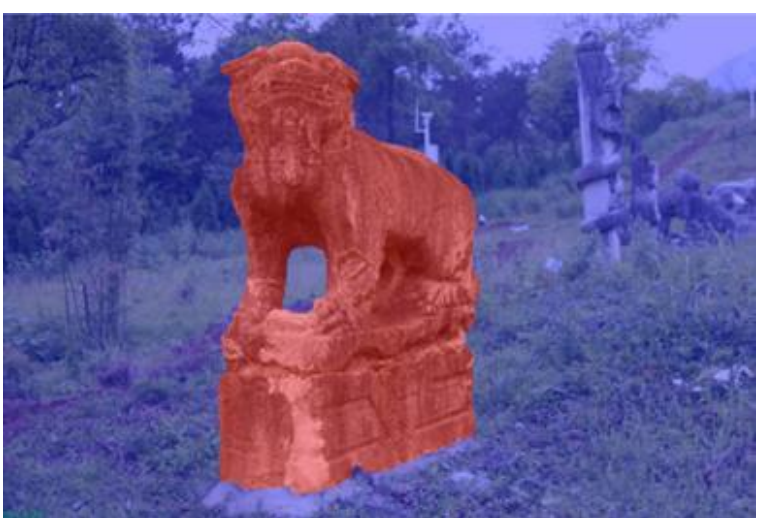

Figure 8. Detected Target Area using One-Cut Algorithm.

\subsection{Texture to Model Matching}

Recovering the spatial position relationship of texture image and geometric model is the precondition of texture mapping. We get the relationship by matching texture image and geometric model. In details, we try to translate and rotate the geometric model in $3 \mathrm{~d}$ space (see in Figure 9), and then perspective projection is used to get the silhouette of model in screen. As introduced in Section 2 , we have already gotten the silhouette of object in texture image, so we can match texture and model by matching their $2 \mathrm{D}$ silhouette information.

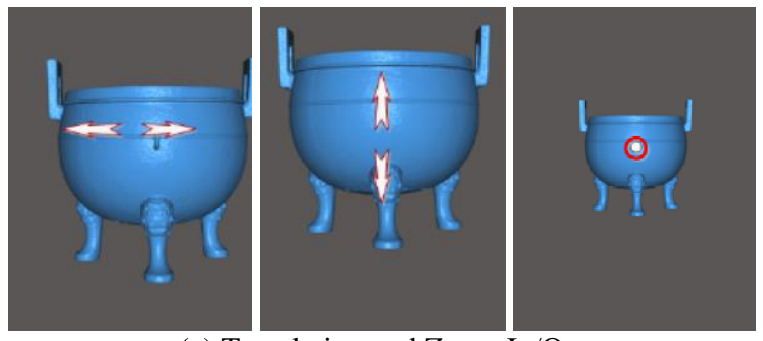

(a) Translation and Zoom In/Out
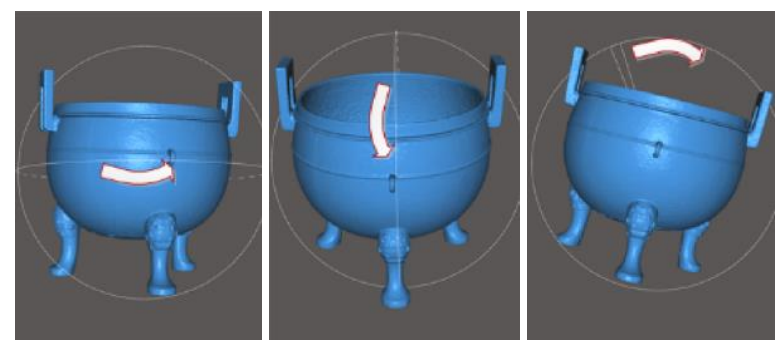

(b) Rotation at Three Axis

Figure 9. The Translation and Rotation of 3D model

\subsubsection{Similarity Measurement}

We present a similarity measure that can be used in texture and model matching: closest point distance. Analogous to iterative closest point, one point set (boundary points), the reference, is kept fixed, while the other one, as the source point set. For each point in the source point set, matching the closest point to the reference point set and measuring their distances. The energy formulation is formulated as:

$$
\begin{gathered}
E=\sum_{i=1}^{n} d_{i} \\
d_{i}=\sqrt{\left(x_{i}-x_{j}\right)^{2}+\left(y_{i}-y_{j}\right)^{2}}
\end{gathered}
$$


Where $\left(x_{i}, y_{i}\right)$ is the point in source point set, $\left(x_{j}, y_{j}\right)$ is the closest point in reference point set, and $d_{i}$ is their distance. $E$ integrates the distance error over all source points. If two silhouette images are similar, the sum of distances error is small. In order to reduce the consumption for searching closest point, kd-tree is used in our method to improve computation efficiency.

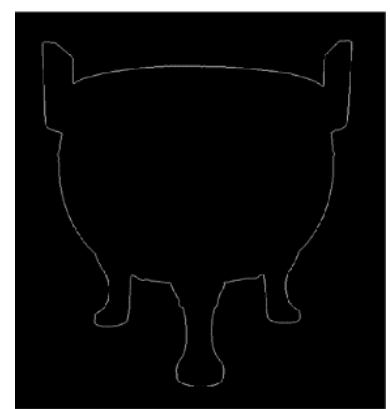

(a)

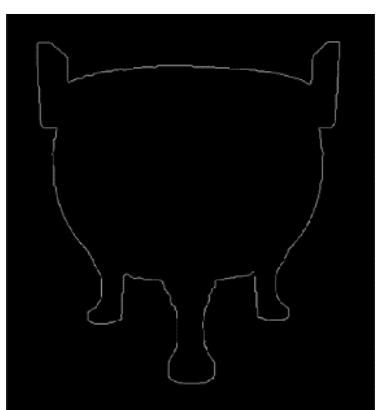

(b)
Figure 10. Silhouette:(a) Silhouette from 3D Model (b) Silhouette from Texture Image

\subsubsection{Parameters Optimization}

To minimize the distance error, the Nelder-Mead simplex algorithm is applied in this our method to optimize the energy function $E$. The Nelder-Mead simplex algorithm is a classical very powerful local descent algorithm, making no use of the objective function derivatives. A "simplex" is a geometrical figure consisting, in $n$ dimensions, of $n+1$ points $x_{1}, x_{2}, \ldots, x_{n}, x_{n+1}$ (Nelder. And Mead, 2003)

To select the appropriate transformation, the method only uses the values of the function to be optimized at the vertices of the simplex considered. After each transformation, the current worst vertex is replaced by a better one.

At the beginning of the algorithm, one moves only the point of the simplex, where the objective function is worst, and one generates an other point image of the worst point. This operation is the reflection. If the reflected point is better than all other points, the method expands the simplex in this direction, otherwise, if it is at least better than the worst, the algorithm performs again the reflection with the new worst point. The contraction step is performed when the worst point is at least as good as the reflected point, in such a way that the simplex adapts itself to the function landscape and finally surrounds the optimum If the worst point is better than the contracted point, the multicontraction is performed. At each step we check that the generated point is not outside the allowed reduced solution space (Pintus et al. 2011). The pseudo code of the algorithm as follows:

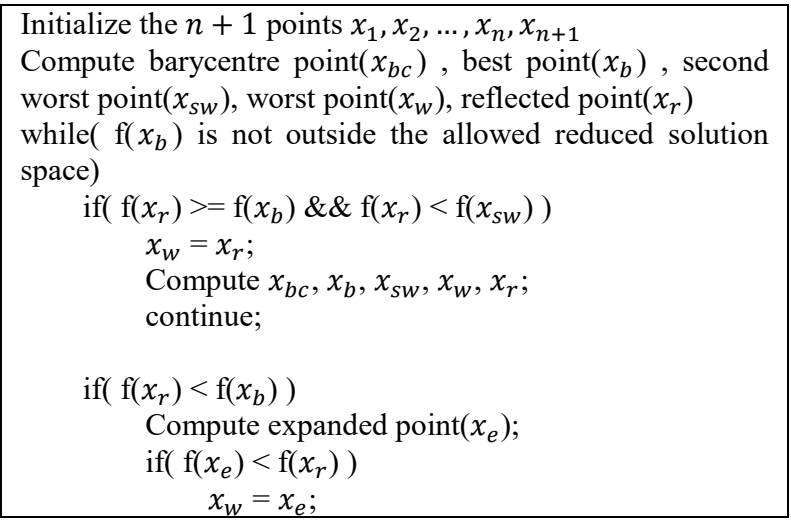

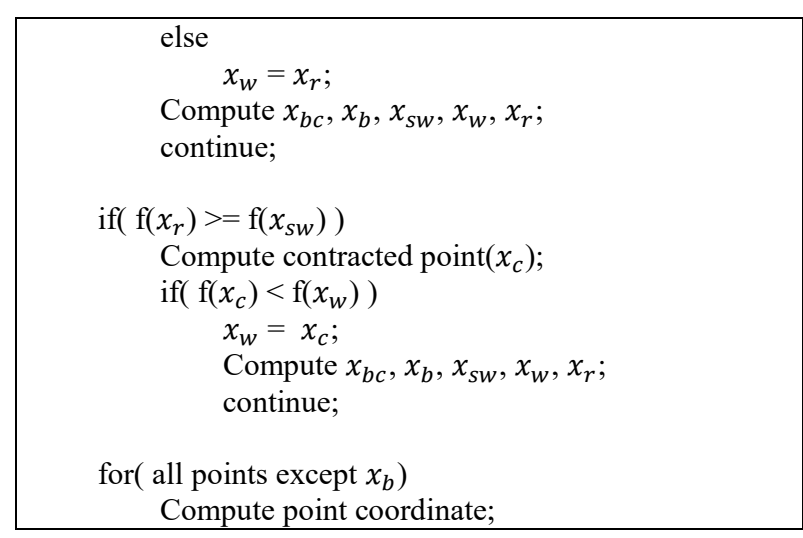

\section{EXPERIMENTS AND CONCLUSION}

\subsection{Test Data}

Data set I: A stone lion sculpture, $3 \mathrm{~d}$ model data contains $100 \mathrm{k}$ triangles, image collected using Canon EOS camera with resolution of $5616 * 3744$ (texture image and geometric model are shown in Figure 1).

Data set II: Bronze tripod from Museum of Wuhan University (in Figure 11), 3d model data contains 1 million triangles, image collect using same camera as data set I.
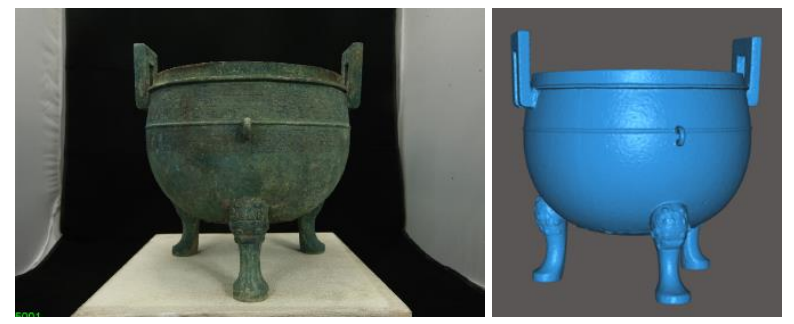

Figure 11: Bronze tripod test dataset.

\subsection{Results}

In this experiment, user only need to set the $3 \mathrm{~d}$ model to the target area in image, without giving precise overlapping. The algorithm automatically finds the target area and mapping texture to $3 \mathrm{~d}$ geometric model.

To reach a proper model to image registration result, the algorithm need an iterative processing to optimize result step-bystep. Since the iterative registration algorithm is sensitive to initial position, we test is in many different distances and view angles. Figure 12 (a) shows the initial position of $3 \mathrm{~d}$ model and image; (b) is the result after geometric model adjustment; (c) is the result of textured model. It is clearly in this figure that the grass area is removed from source image. Only the target area is left using as texture.
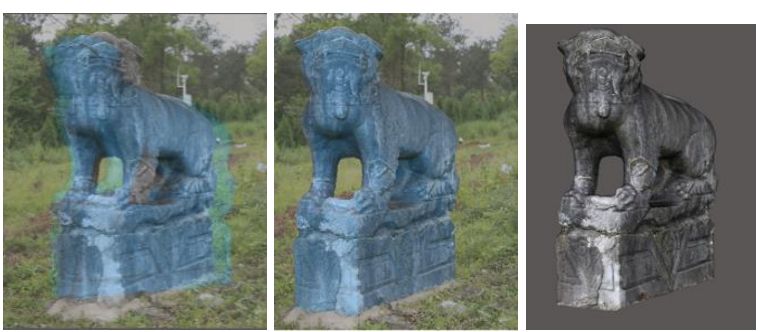


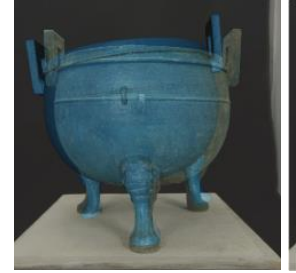

(a)

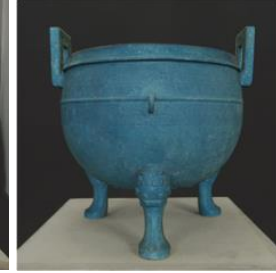

(b)

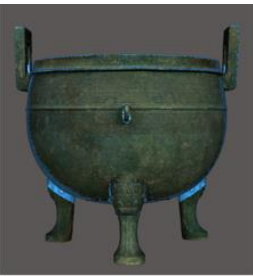

(c)
Figure 12: Results of Target Extraction and Texture Mapping.

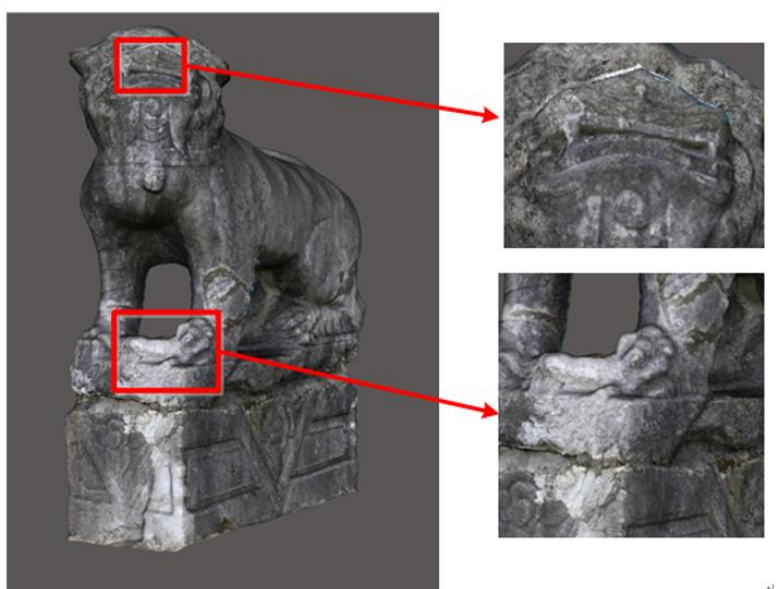

(a) Detailed Texture Mapping Results of Stone Lion.

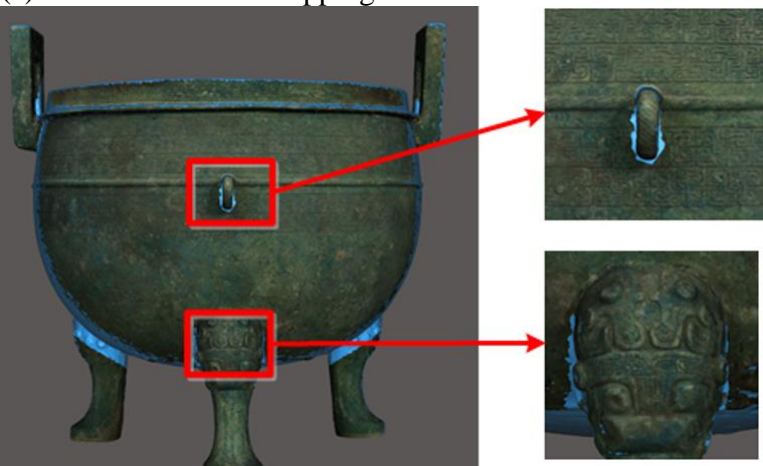

(b) Detailed Texture Mapping Results of Bronze Tripod. Figure 13: Details ofTexture Mapping Result.

\subsection{Robustness Analysis}

The robustness of this algorithm is another factor we evaluate in this experiment. For each test dataset, we select 64 different initial positions. Two indicators are measured to evaluate the processing speed and registration quality, which are times of iteration and registration error.

Figure 14 is the robustness analysis results of stone lion. Blue line is the iterative time (number at left side) for each initial position. Red line shows registration error after many times of iteration. Only one failed in 64 giving initial positions. Figure 15 is the test results of bronze tripod. In test dataset II, all the 64 texture mapping test successfully converge to a stable state, resulting to good registration quality.

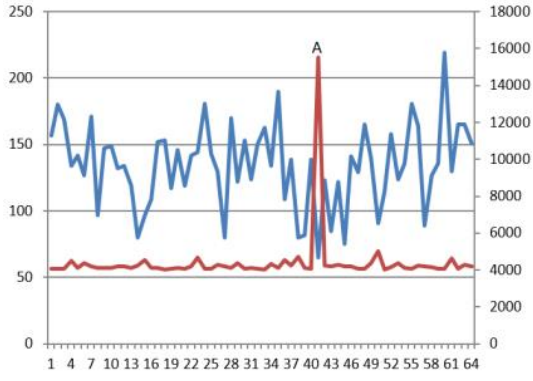

Figure 14: Details ofTexture Mapping Result.

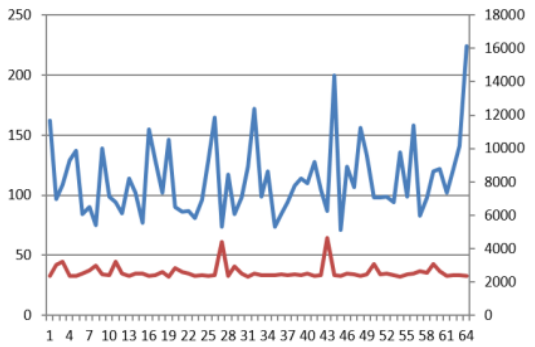

Figure 15: Details ofTexture Mapping Result.

From these two groups of experiments, we can find that: though the initial positions are different, the final registration errors are almost same after the iteration processing.

We also extend this algorithm and integrate this algorithm into a software named ModelPaitner. Matching image to $3 \mathrm{~d}$ geometric model is the first step for high fidelity texture modeling. What beyond matching are color adjustment, best image selection, texture seam processing, etc. Each of these work is very time consuming. ModelPainter is a software we developed specially for texture processing, especially for culture heritage modeling.

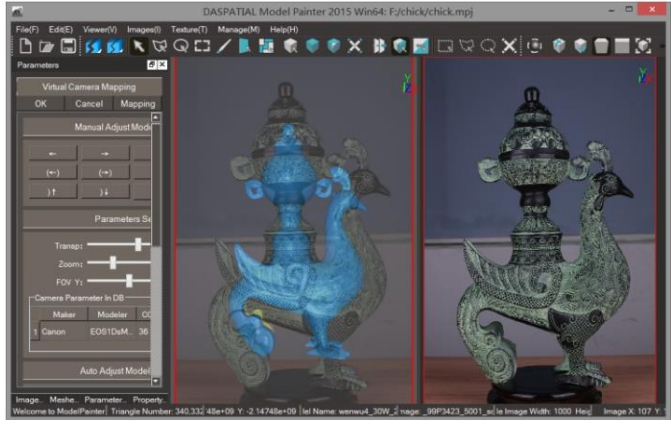

(a) . UI of ModelPainter Software

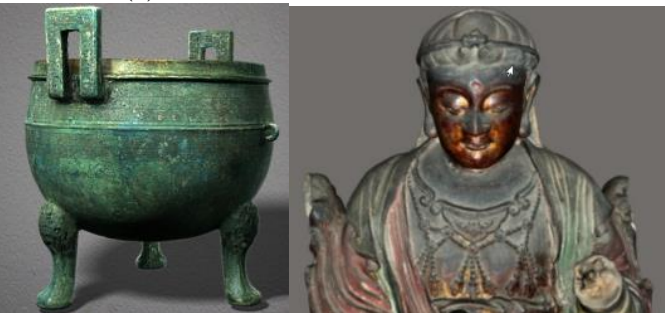

(b). 3D Textured Model Build by ModelPainter.

Figure 16: ModelPainter UI and the Produced 3D Model Data.

\subsection{Conclusion}

High fidelity texture mapping is an important work for heritage modelling. In this, we propose a semi-automatic image to $3 \mathrm{~d}$ model registration algorithm, which is able detect target object and mapping texture to geometric model using detected 
silhouette information. Experiments using data from real project proved the algorithm we proposed.

The benefit of this method includes: (1) image to model registration result is stable and insensitive to initial position; (2) only need very simple user interaction; (3) high efficiency.

This method works well on single image to model registration. If the texture images have abundant texture information enough for aerial-triangulation, mapping image to model according exterior parameters of image will be a proper solution. Currently, we didn't add multi-image global optimization into the algorithm, which might be done in the future.

\section{ACKNOWLEDGEMENTS}

This research was supported by the National Key Technology Research and Development Program of China No. 2014BAK07B04, National Natural Science Foundation of China No. 41571437, Funding of YunGang Grottoes.

\section{REFERENCES}

Lensch, H.P., Heidrich, W. and Seidel, H.P., 2001. A silhouettebased algorithm for texture registration and stitching. Graphical Models, 63(4), pp.245-262.

Fontana R., Greco M., Materazzi M., Pampaloni E., Pezzati L., Rocchini C., Scopigno R., 2002. Three-dimensional modelling of statues: the Minerva of Arezzo, Journal of Cultural Heritage, 3 pp, 325-331.

Ikeuchi K., Oishi T., Takamatsu J., Sagawa R., Nakazawa A., Kurazume R., Nishino K., Kamakura M., Okamoto Y., 2007. The Great Buddha Project: Digitally Archiving, Restoring, and Analyzing Cultural Heritage Objects, Int. J. Comput. Vision, 75, pp. 189-208.

Pavlidis G., Koutsoudis A., Arnaoutoglou F., Tsioukas V., Chamzas C., 2007. Methods for 3D digitization of Cultural Heritage, Journal of Cultural Heritage, 8, pp. 93-98.

Chow S.-K., Chan K.-L., 2009. Reconstruction of photorealistic 3D model of ceramic artefacts for interactive virtual exhibition, Journal of Cultural Heritage, 10, pp. 161-173.

Arbace L., Sonnino E., M. Callieri, M. Dellepiane, M. Fabbri, A. Iaccarino Idelson, R. Scopigno. 2012. Innovative uses of 3D digital technologies to assist the restoration of a fragmented terracotta statue, Journal of Cultural Heritage, http://dx.doi.org/10.1016/j.culher.

Andrade B. T., Bellon O.R.P., Silva L., Vrubel A., 2012. Digital preservation of Brazilian indigenous artworks: Generating high quality textures for 3D models, Journal of Cultural Heritage, 13, pp. 28-39.

Tzur Y., Tal A., 2009. FlexiStickers - Photogrammetric Texture Mapping using Casual Images, SIGGRAPH 2009, ACM Transactions on Graphics, 28. pp. 1-10.

Nelder J.A., Mead R., 1965. A simplex method for function minimization, The Computer Journal. 7, pp. 308-313.

Chelouah R., Siarry P., 2003, Genetic and Nelder-Mead algorithms hybridized for a more accurate global optimization of continuous multiminima functions, European Journal of Operational Research, 148(2), pp. 335-348.

Pintus R, Gobbetti E, Combet R. 2011, Fast and Robust SemiAutomatic Registration of Photographs to 3D Geometry. International Symposium on Virtual Reality Archaeology \& Cultural Heritage, 7(4), pp.9-16.

Boykov Y Y, Jolly M P. 2001. Interactive graph cuts for optimal boundary \& region segmentation of objects in N-D images[C]. ICCV 2001. Proceedings. Eighth IEEE International Conference on. IEEE:105 - 112. 